\title{
EQUAÇÃO DE LONGEVIDADE PARA SEMENTES DE CAFÉ (Coffea arabica L.)
}

\author{
Roberto Sinício ${ }^{1}$
}

\section{RESUMO}

Objetivou-se equacionar a longevidade de sementes de café usando o Modelo de Probit. Valores obtidos da literatura de curvas de sobrevivência de sementes de café, armazenadas sob diferentes temperaturas e teores de água, foram transformados em probit e utilizados em regressões lineares e não lineares para determinação dos coeficientes de equações de viabilidade. Os resultados das análises permitiram concluir que: um modelo quadrático apresentou ajuste adequado, com desvio padrão de $8,9 \%$ de germinação, para equacionar a perda de germinação de sementes de café armazenadas, enquanto o modelo tradicional apresentou desvio padrão de 15,1\% de germinação; a regressão não linear, comparada com a regressão linear múltipla, resultou em menores 1,5 a 2,2 vezes desvios padrão na determinação dos coeficientes da equação do desvio padrão da frequência de distribuição das sementes mortas durante o período de armazenamento $(\sigma)$; e as sementes de café não apresentaram comportamento de ortodoxas, indicado pelas variações não lineares do logaritmo de $\sigma$ em função das variações do logaritmo do teor de água das sementes.

Palavras-chave: Armazenamento, Germinação, Modelo de Probit, Modelo Matemático, Previsão.

\section{ABSTRACT \\ LONGEVITY EQUATION FOR COFFEE SEEDS (Coffea arabica L.)}

The objective of this study was to develop an equation to estimate longevity of coffee seeds using the Probit Model. Published survival curves of coffee seeds, stored under different temperatures and moisture contents, were transformed to probit and used in linear and non-linear multiple regressions to determine the coefficients of viability equations. The results showed adequate fitting in a quadratic model with a standard deviation of $8.9 \%$ germination, compared to $15.1 \%$ in the traditional model. The non-linear regression compared to the multiple linear regression, gave lower ( 1.5 to 2.2 times) standard deviation to determine the coefficient of the equation of the standard deviation of distribution frequency of dead seed during the storage period $(\sigma)$. The coffee seeds did not behave as orthodox seeds, as indicated by the non linear variations of the logarithm of $\sigma$ as a function of the variations of the logarithm of the seed moisture content.

Keywords: Germination, Mathematical Model, Prediction, Probit Model, Storage.

Recebido para publicação em 18/11/2009. Aprovado em 24/02/2011.

1- Engenheiro de Alimentos, Ph. D., Pesquisador, Departamento de Fitotecnia-UFV, e-mail: rsinicio@ufv.br 


\section{INTRODUÇÃOO}

A maioria do germoplasma é armazenada como semente nos centros de recursos genéticos nacionais e internacionais. Via de regra, a responsabilidade dos bancos de germoplasma é armazenar sementes em longo prazo, por mais de cinco anos, e com a manutenção da viabilidade mais alta possível. A manutenção dessa diversidade genética é uma preocupação mundial (VERTUCCI \& ROSS, 1990), pois é extremamente importante para futuras práticas de melhoramento vegetal, particularmente em relação à resistência a doenças, estresse e produtividade (BEWLEY \& BLACK, 1994).

A previsão da perda de viabilidade de sementes armazenadas por meio de modelos matemáticos é importante tanto para a manutenção de bancos de germoplasma como para o gerenciamento da produção e armazenamento de sementes (MEAD \& GRAY, 1999). A necessidade de monitoramento das sementes armazenadas é diminuída na medida em que são desenvolvidos modelos matemáticos com maior precisão para predizer a perda de viabilidade. O monitoramento da viabilidade é a etapa que exige maior demanda de mão de obra para a manutenção de bancos de germoplasma (BEWLEY \& BLACK, 1994).

Em estudos de conservação de sementes, devese levar em consideração o seu comportamento fisiológico em relação ao armazenamento, a fim de manter nível de qualidade delas, sendo que a capacidade de armazenamento varia entre as espécies, bem como entre e dentro dos lotes de sementes da mesma espécie (GROOT et al., 2003). Vários são os fatores que influenciam a conservação da viabilidade e do vigor das sementes durante o armazenamento, entre eles: qualidade fisiológica inicial das sementes, vigor da planta mãe, condições climáticas durante a maturação, danos mecânicos, condições de secagem, adequado teor de água, umidade relativa do ar, temperatura de armazenamento, ação de fungos e insetos, tipos de embalagens e tempo de armazenamento (CARVALHO \& NAKAGAWA, 2000).

O modelo matemático de Probit, descrito por Ellis \& Roberts (1980) para previsão da perda de germinação, é utilizado para estabelecer as recomendações de armazenagem comercial em bancos de germoplasma. Neste modelo, entretanto, para uma mesma condição ambiental, existe uma resposta diferenciada em relação ao armazenamento para cada espécie. Logo, há necessidade de serem determinados coeficientes para cada espécie de semente armazenada. No modelo de Ellis \& Roberts (1980), a curva de sobrevivência das sementes ortodoxas pode ser descrita por:

$$
\begin{aligned}
& v=K i-p / \sigma \\
& \sigma=10^{\left(K E-C W \log m-C H t-C Q t^{2}\right)}
\end{aligned}
$$

em que,

$v=$ viabilidade das sementes (probit);

$K i=$ viabilidade inicial das sementes (probit);

$p=$ período de armazenamento (dias);

$\sigma=$ desvio padrão da frequência de distribuição das sementes mortas durante o período de armazenamento (dias);

$m=$ teor de água ( $\%$, base úmida);

$t=$ temperatura $\left({ }^{\circ} \mathrm{C}\right)$; e

$K E, C W, C H$ e $C Q=$ coeficientes específicos para cada espécie, independentemente do genótipo e das condições de pré-estocagem.

A perda da germinação de sementes armazenadas tem sido prevista com sucesso para muitas espécies ortodoxas, utilizando-se o modelo de Ellis \& Roberts (1980). Os coeficientes $K E, C W, C H$ e $C Q$ deste modelo já foram determinados, por diferentes pesquisadores, para mais de 77 cultivares de 48 espécies (ELLIS \& ROBERTS, 1980; ELLIS et al., 1982; HONG et al., 1996; USBERTI \& GOMES, 1998; SINÍCIO et al., 2008; SINÍCIO et al., 2009).

$K i$ é uma constante que representa uma estimativa da qualidade inicial das sementes, específica para cada lote de sementes, a qual depende de vários fatores, sendo os mais importantes o genótipo, as condições de pré-armazenamento e a sua interação (ELLIS \& ROBERTS, 1980). Para determinar $\mathrm{Ki}$ com maior precisão, Ellis \& Roberts (1980) sugerem que essa determinação seja feita por meio do teste de envelhecimento acelerado, no qual as sementes são rapidamente deterioradas, sob condições constantes e adversas de umidade e temperatura, em um determinado período de tempo. Testes de germinação 
sucessivos seriam realizados com amostras retiradas em intervalos regulares nas condições acima citadas, calculando-se assim a curva de sobrevivência das sementes por meio da análise de probit (FINNEY, 1971). O valor de $K i$ poderia ser determinado, na interceptação da curva de sobrevivência das sementes plotada em probit, com o tempo zero de armazenamento.

O modelo de probit tem grande aplicação na produção comercial de sementes e no gerenciamento de bancos de germoplasma, apesar de algumas inadequações deste modelo, relacionadas com a determinação de $K i$, terem sido apontadas por Fabrizius et al. (1999) e Mead \& Gray (1999).

Considerando-se que não foi encontrado na literatura nenhum estudo sobre a longevidade de sementes de café, objetivou-se com este trabalho equacionar a perda da germinação de sementes de café armazenadas, utilizando o modelo de probit.

\section{MATERIAL E MÉTODOS}

Os dados experimentais de sementes de café armazenadas obtidos por Gentil et al. (2001) foram utilizados para o equacionamento da longevidade. Esses dados contêm as perdas de germinação de sementes de café acondicionadas em sacos de polietileno e armazenadas durante 48 semanas em condições constantes de temperatura $\left(10,20\right.$ e $\left.30^{\circ} \mathrm{C}\right)$ e de teor de água $(10,16,23,34,41$ e 51\%). Durante o armazenamento, foram realizadas avaliações periódicas do teor de água, germinação, vigor (comprimento de plântula, massa de matéria seca e velocidade de emergência de plântulas) e sanidade das sementes do café. GENTIL et al. (2001) constataram que as reduções do teor de água até $10 \%$ e da temperatura até $10^{\circ} \mathrm{C}$ são favoráveis à manutenção da qualidade fisiológica das sementes e que teor de água próximo a $23 \%$ favorece o estabelecimento de Penicillium sp. e de Aspergillus sp. nas sementes.

Os valores de percentagem de germinação das sementes armazenadas foram, primeiramente, transformados em probit, de modo que seus ciclos de vida ficassem uniformemente distribuídos (ELLIS \& ROBERTS, 1980). Posteriormente, esses dados foram utilizados em análises estatísticas para estimativa dos coeficientes de diferentes equações de viabilidade. Nestas análises, foram realizadas regressões lineares simples e múltiplas (Programa Excel da Microsoft) e regressões não lineares (PRESS et al., 1990).

Os valores dos coeficientes da equação de $\sigma$ foram determinados utilizando as seguintes metodologias: 1) Regressões lineares múltiplas (método tradicional) e regressões não-lineares, com base nos valores de $\sigma$ determinados para as curvas de sobrevivência nas diferentes temperaturas e teores de água das sementes armazenadas; e 2) Regressões não lineares com base nos valores da perda de germinação, $K i$, temperatura, teor de água e período de armazenagem, para todas as curvas de sobrevivência das sementes.

$\mathrm{Na}$ primeira metodologia, foram determinados os valores de $K i$ e $\sigma$ (Equação 1) por meio de regressões lineares simples, para cada combinação de temperatura e teor de água das sementes armazenadas em condições controladas (o valor de $\sigma$ é igual ao negativo do inverso do coeficiente angular da reta). Em seguida, os coeficientes das equações de $\sigma$ foram determinados por meio de regressões lineares múltiplas e regressões não lineares em função do teor de água e da temperatura. A equação de $\sigma$ (Equação 2) foi linearizada com uso de logaritmos para realização das regressões lineares múltiplas.

$\mathrm{Na}$ segunda metodologia, os coeficientes das equações de $\sigma$ foram determinados por meio de regressões não lineares para todas as curvas de sobrevivência, simultaneamente, com base nos valores da perda de germinação, das médias de $K i$, da temperatura, do teor de água e do período de armazenagem (Equações 1 e 2). As médias de Ki foram calculadas para os seis níveis de teor de água inicial.

Vários modelos foram testados por meio de regressões lineares e não lineares com a finalidade de determinar os coeficientes das equações de $\sigma$, observando-se parâmetros estatísticos tais como o coeficiente de regressão ajustado $\left(\mathrm{R}^{2}\right)$, desvio padrão e nível de significância estatística dos coeficientes pelo teste $t$.

\section{RESULTADOS E DISCUSSÃO}

$\mathrm{O} \mathrm{R}^{2}$ médio de 0,6443 e o desvio padrão médio de 0,4308 probit indicam que o Modelo de Probit não apresentou alta precisão no ajuste para sementes de café (Quadro 1). O desvio padrão médio de $K i$ 
obtido é bem maior do que $0,16 \pm 0,06$ probit obtido por Pieta Filho et al. (1992) em pesquisa objetivando determinar $K i$ para seis lotes de cevada e oito lotes de trigo, sendo semelhante a $0,46 \pm 0,31$ probit obtido por FABRIZIUS et al. (1999) para 12 lotes de soja.

$\mathrm{O}$ menor desvio padrão (teste $4, \mathrm{R}^{2}$ de 0,935 e d.p. de 78,2 dias) foi obtido por meio de regressão não linear (Quadro 2). Observa-se também que os coeficientes $C H$ e $C Q$ da equação proposta por Ellis $\&$ Roberts (1980) não foram significativos (Quadro 2).

As equações obtidas nos testes de 1 a 4 (Quadro 2), quando utilizadas para calcular a perda de germinação de sementes de café armazenadas (Equação 1), resultaram em $\mathrm{R}^{2}$ de $0,512,0,330,0,655$ e 0,685 e desvios padrão de 17,7,28,7,15,8 e 14,0\% de germinação, respectivamente. Sobre a baixa precisão obtida no ajuste da equação proposta por Ellis \& Roberts (1980) para café, Ellis et al. (1990) e Hong \& Ellis (1996) comentam que as sementes de cafeeiro deveriam ser incluídas na categoria intermediária da classificação de Roberts (1973). Assim, seria admitido que as sementes "ortodoxas" toleram dessecação a teores de água próximos de 5\%, "intermediárias" toleram dessecação a teores de água em torno de $10-12,5 \%$ e têm a viabilidade reduzida em teores de água inferiores e as "recalcitrantes" perdem a viabilidade quando dessecadas a $15-20 \%$ de teor de água.

É interessante observar que a regressão não linear, comparada com a regressão linear múltipla, resultou em menores desvios padrão na determinação dos coeficientes das equações de $\sigma$ (testes de 1 a 4 , Quadro 2). SAPRA et al. (2003) explicam que a equação de $\sigma$, linerizada com o uso de logaritmos, apresenta distribuição simétrica de erros para o log $\sigma$, mas assimétrica para $\sigma$, dando uma estimativa da mediana da distribuição dos erros e não da média dos erros.

As variações do logaritmo de $\sigma$ em função das variações do logaritmo do teor de água e da temperatura, calculadas pelas equações dos testes $1 \mathrm{e}$ 4 (Figura 1 e Quadro 2), mostram o comportamento das sementes de café durante o armazenamento em relação ao grau de dessecação no modelo de probit. Observa-se que as sementes de café apresentaram comportamento não linear do logaritmo de $\sigma$ em relação ao logaritmo do teor de água, fato este não observado para a equação utilizada por Ellis \& Roberts (1980) para sementes ortodoxas.

Quadro 1. Regressões lineares das curvas de sobrevivência das sementes de café armazenadas sob diferentes temperaturas e teores de água.

\begin{tabular}{ccccccc}
\hline Teste & $\begin{array}{c}\text { Temperatura } \\
\left({ }^{\circ} \mathrm{C}\right)\end{array}$ & $\begin{array}{c}\text { Teor de água } \\
(\%, \text { b.u. })\end{array}$ & $\begin{array}{c}\mathrm{Ki} \\
(\text { probit })\end{array}$ & $\begin{array}{c}\sigma \\
(\text { dias })\end{array}$ & $\begin{array}{c}\mathrm{R}^{2} \\
(\text { decimal) }\end{array}$ & $\begin{array}{c}\text { Desvio Padrão } \\
\text { (probit) }\end{array}$ \\
\hline 1 & 30 & 34,63 & 1,9815 & 40,15 & 0,8374 & 0,5932 \\
2 & 30 & 10,20 & 1,1797 & 71,95 & 0,9098 & 0,2393 \\
3 & 20 & 51,52 & 1,5901 & 49,77 & 0,8951 & 0,3752 \\
4 & 20 & 41,59 & 1,6014 & 199,17 & 0,5703 & 0,3041 \\
5 & 20 & 34,43 & 2,3273 & 96,92 & 0,6562 & 0,8037 \\
6 & 20 & 22,54 & 1,7857 & 58,99 & 0,6438 & 0,7834 \\
7 & 20 & 15,93 & 1,7707 & 96,15 & 0,4506 & 0,8828 \\
8 & 20 & 10,49 & 1,1256 & 716,53 & 0,4952 & 0,1482 \\
9 & 10 & 51,69 & 1,6408 & 113,53 & 0,8804 & 0,3561 \\
10 & 10 & 41,75 & 1,9474 & 165,67 & 0,7336 & 0,3945 \\
11 & 10 & 34,45 & 1,8435 & 185,60 & 0,8947 & 0,2029 \\
12 & 10 & 22,61 & 1,2116 & 577,70 & 0,1861 & 0,3334 \\
13 & 10 & 16,25 & 1,2321 & 968,79 & 0,2222 & 0,1835 \\
\hline Média & - & - & 1,6336 & 256,99 & 0,6443 & 0,4308 \\
\hline
\end{tabular}


Quadro 2. Equações obtidas para calcular $\sigma$ em função do teor de água e da temperatura de armazenamento das sementes de café usando regressões lineares (L) e não lineares (NL)

\begin{tabular}{|c|c|c|c|c|}
\hline Teste & Regressão & Equação & $\begin{array}{c}\mathrm{R}^{2} \\
(\text { decimal) }\end{array}$ & $\begin{array}{l}\text { Desvio Padrão } \\
\text { (dias) }\end{array}$ \\
\hline 1 & $\mathrm{~L}$ & 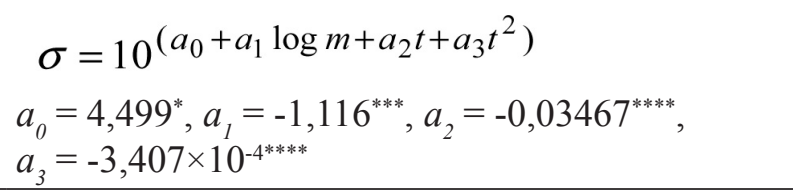 & 0,793 & 201,9 \\
\hline 2 & NL & 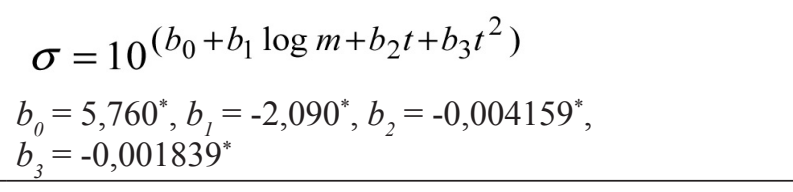 & 0,906 & 93,7 \\
\hline 3 & $\mathrm{~L}$ & 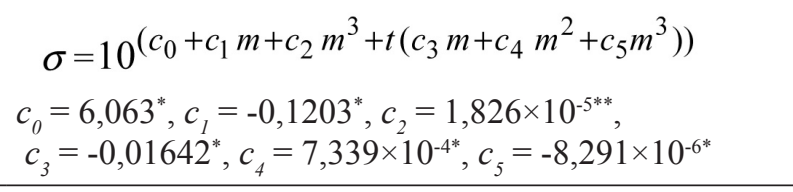 & 0,903 & 113,7 \\
\hline 4 & NL & $\begin{array}{l}\sigma=10^{\left(d_{0}+d_{1} m+d_{2} m^{3}+t\left(d_{3} m+d_{4} m^{2}+d_{5} m^{3}\right)\right)} d_{0} \\
=5,120^{*}, d_{1}=-0,08377^{*}, d_{2}=9,951 \times 10^{-6^{*}} \\
d_{3}=-0,01143^{*}, d_{4}=4,924 \times 10^{-4 *}, d_{5}=-5,355 \times 10^{-6^{*}}\end{array}$ & 0,935 & 78,2 \\
\hline
\end{tabular}

*Significativo em nível de $0,1 \%$ de probabilidade de erro no teste $t$

** Significativo em nível de $1,0 \%$ de probabilidade de erro no teste $t$

*** Significativo em nível de 2,0\% de probabilidade de erro no teste $t$

${ }^{* * * *}$ Não significativo em nível de $60,0 \%$ de probabilidade de erro no teste $t$

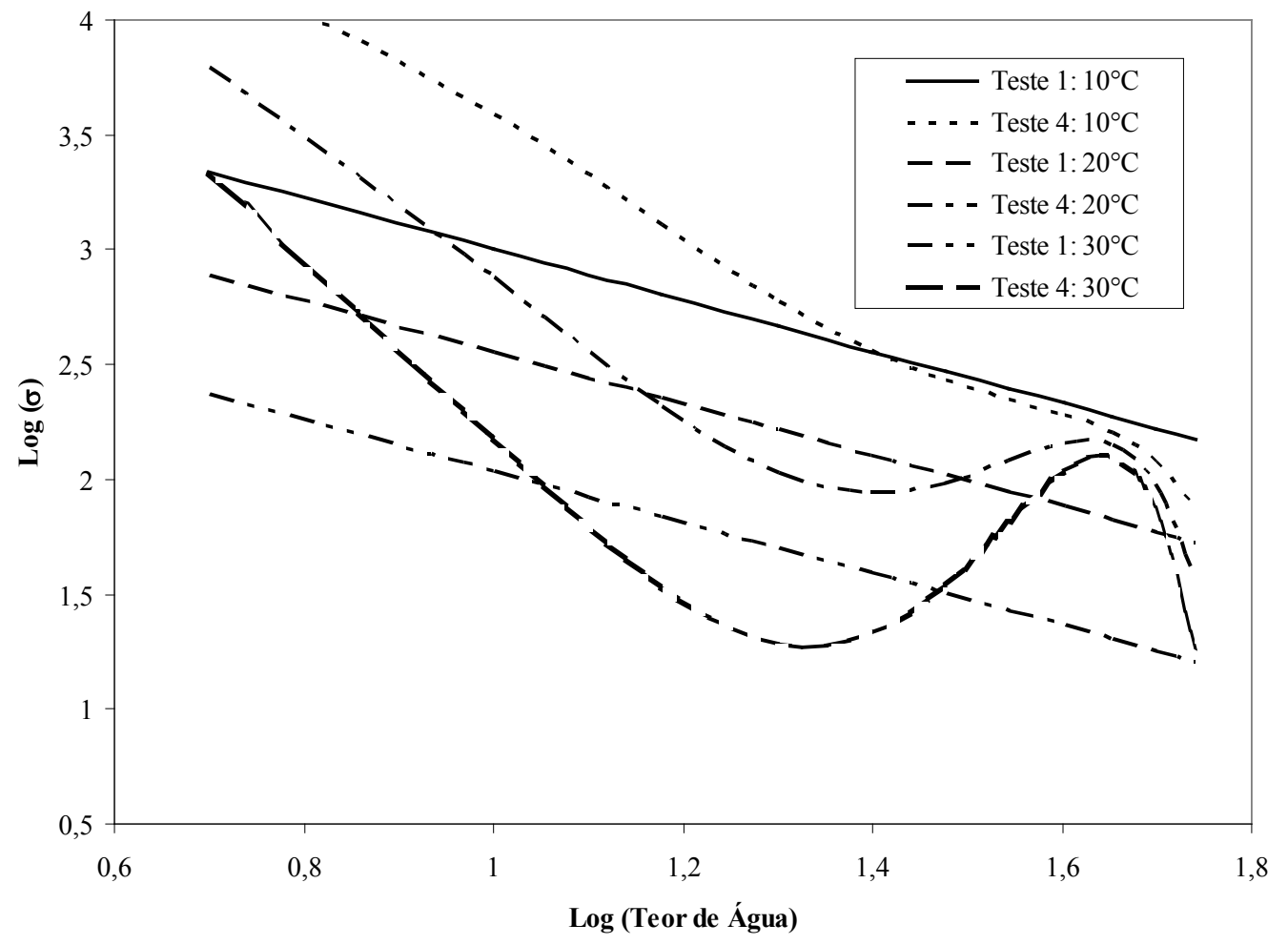

Figura 1. Logaritmo de em função do logaritmo do teor de água para sementes de café nas temperaturas de 10,20 e $30^{\circ} \mathrm{C}$ e teores de água variando de 5 a $55 \%$. 
Quadro 3. Equações obtidas usando regressões não lineares para calcular a perda de germinação de sementes de café armazenadas em função das médias de Ki, do período, do teor de água e da temperatura de armazenamento para todas as curvas de sobrevivência

\begin{tabular}{|c|c|c|c|}
\hline Teste & Equação & $\begin{array}{c}\mathrm{R}^{2} \\
\text { (decimal) }\end{array}$ & $\begin{array}{c}\text { Desvio Padrão } \\
(\%)\end{array}$ \\
\hline 1 & 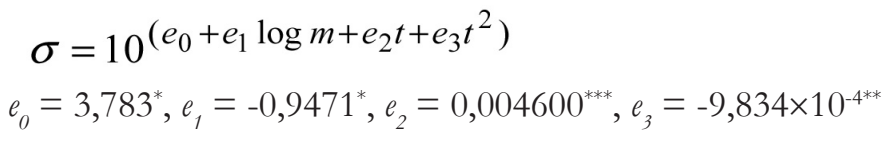 & 0,621 & 15,1 \\
\hline 2 & 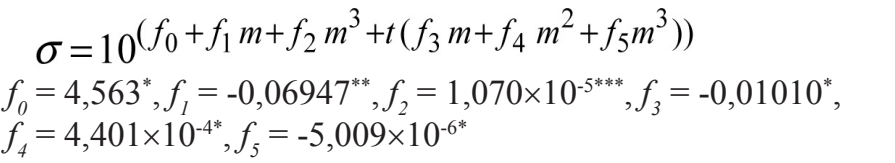 & 0,741 & 12,5 \\
\hline
\end{tabular}

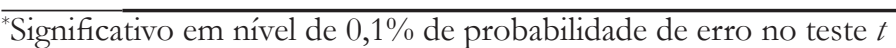

*Significativo em nível de 1,0\% de probabilidade de erro no teste $t$

${ }^{*}$ Significativo em nível de $10,0 \%$ de probabilidade de erro no teste $t$

Melhor resultado para calcular a perda de germinação, entretanto, foi obtido usando regressão não linear na determinação dos coeficientes das equações de $\sigma$ para todas as curvas de sobrevivência, simultaneamente, com base nos valores da perda de germinação, das médias de $K i$, da temperatura, do teor de água e do período de armazenagem, para um total de 130 observações experimentais (teste 2, Quadro 3). As médias de $K i$ foram 1,0416, 1,5014, 1,4986, 2,0508, 1,7744 e 1,6154, respectivamente, para os teores de água médios de 10,20, 15,93, 22,54, 34,63, 41,59 e $51,52 \%$.

Os resultados das análises de regressão não resultaram em ajustes satisfatórios do modelo de probit (Equação 1) para predizer a perda de germinação de sementes de café armazenadas. Um modelo quadrático de probit, comparado ao modelo linear, se ajustou bem melhor aos dados das curvas de sobrevivência das sementes, resultando num $\mathrm{R}^{2}$ médio de 0,7351 e num desvio padrão médio de 0,2938 probit. Por isso, decidiu-se testar o seguinte modelo:

$$
\begin{aligned}
& v=K-p / \sigma_{1}-p^{2} / \sigma_{2} \\
& \sigma_{1}=10^{\left(g_{0}+g_{1} m+t\left(g_{2} m+g_{3} m^{3}\right)\right)}
\end{aligned}
$$

em que,

$$
\begin{aligned}
& g_{0}=15,47^{*}, g_{1}=-0,2783^{* *} \text {, } \\
& g_{2}=-9,421 \times 10^{-3 * *} \text {, } \\
& g_{3}=4,537 \times 10^{-6^{* * * *}} \\
& \sigma_{2}=10^{\left(h_{0}+h_{1} m+h_{2} m^{3}+t\left(h_{3} m+h_{4} m^{2}+h_{5} m^{3}\right)\right)}
\end{aligned}
$$

sendo que,

$h_{0}=9,525^{*}, h_{1}=-0,1845^{* *}, h_{2}=4,086 \times 10^{-5 * * *}, h_{3}$ $=-0,02106^{*}, h_{4}=1,045 \times 10^{-3^{*}}$ e $h_{5}=-1,303 \times 10^{-5^{*}}$ ( ${ }^{*}$ Significativo em nível de $0,1 \%$, ${ }^{*}$ significativo em nível de $0,5 \%$ e ** significativo em nível de $1,0 \%$ de probabilidade de erro no teste $t$ ).

Os coeficientes de $\sigma_{1}$ e $\sigma_{2}$ foram determinados utilizando regressões não lineares para todas as curvas de sobrevivência, simultaneamente, com base nos valores da perda de germinação, das médias de $K i$, da temperatura, do teor de água e do período de armazenagem, para 130 observações experimentais. Esse modelo apresentou um $\mathrm{R}^{2}$ de 0,878 e um desvio padrão de $8,9 \%$ de germinação. Os gráficos dos resultados calculados e experimentais da perda de germinação do café armazenado (Figuras 2, 3 e 4) mostram a precisão do ajuste deste modelo aos dados de Gentil et al. (2001). 


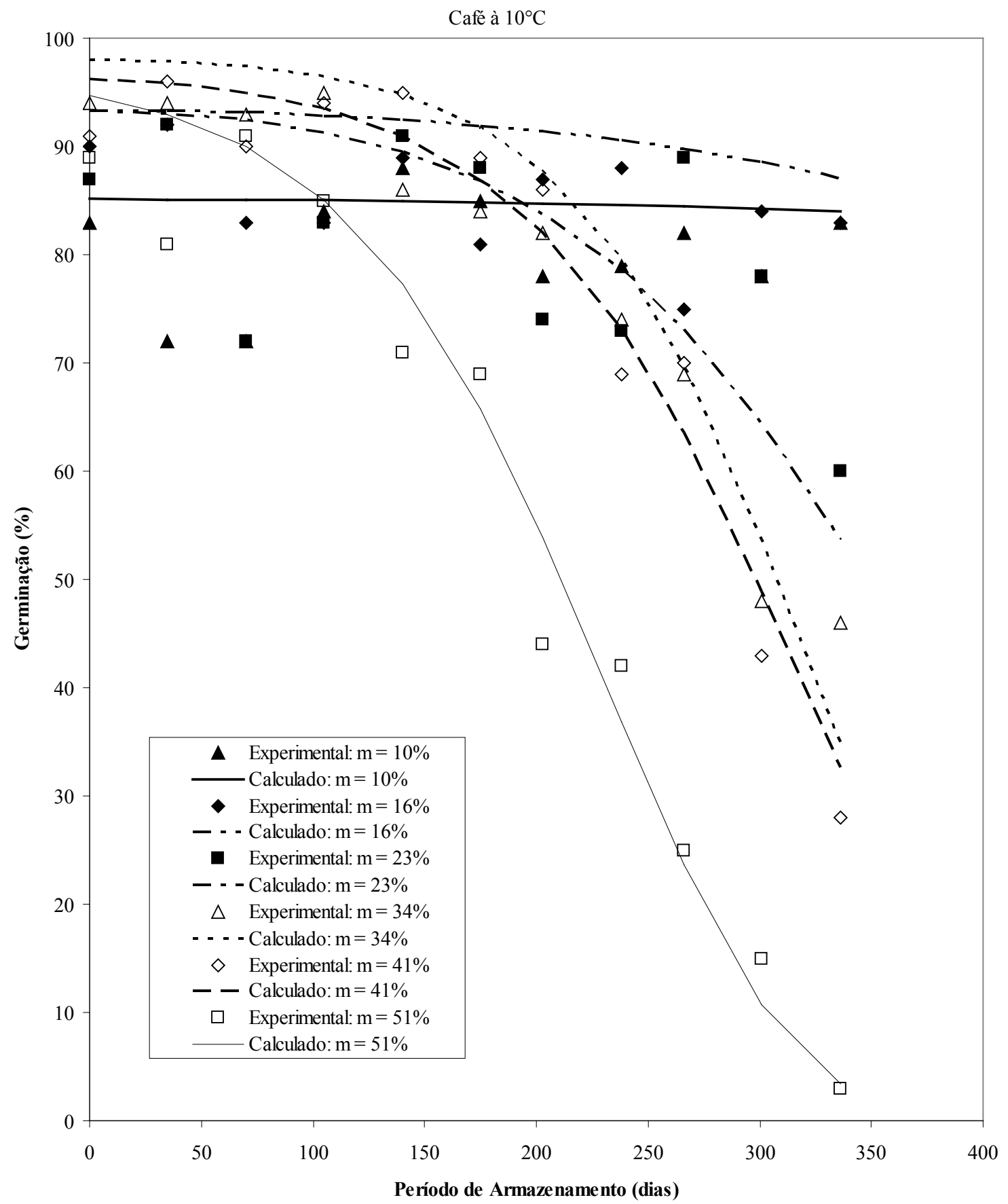

Figura 2. Valores experimentais e calculados (modelo proposto) da perda de germinação das sementes de café em função do período de armazenamento na temperatura de $10^{\circ} \mathrm{C}$ e teores de água de 10,16 , 23, 34, 41 e $51 \%$ (dados experimentais de Gentil et al., 2001). 


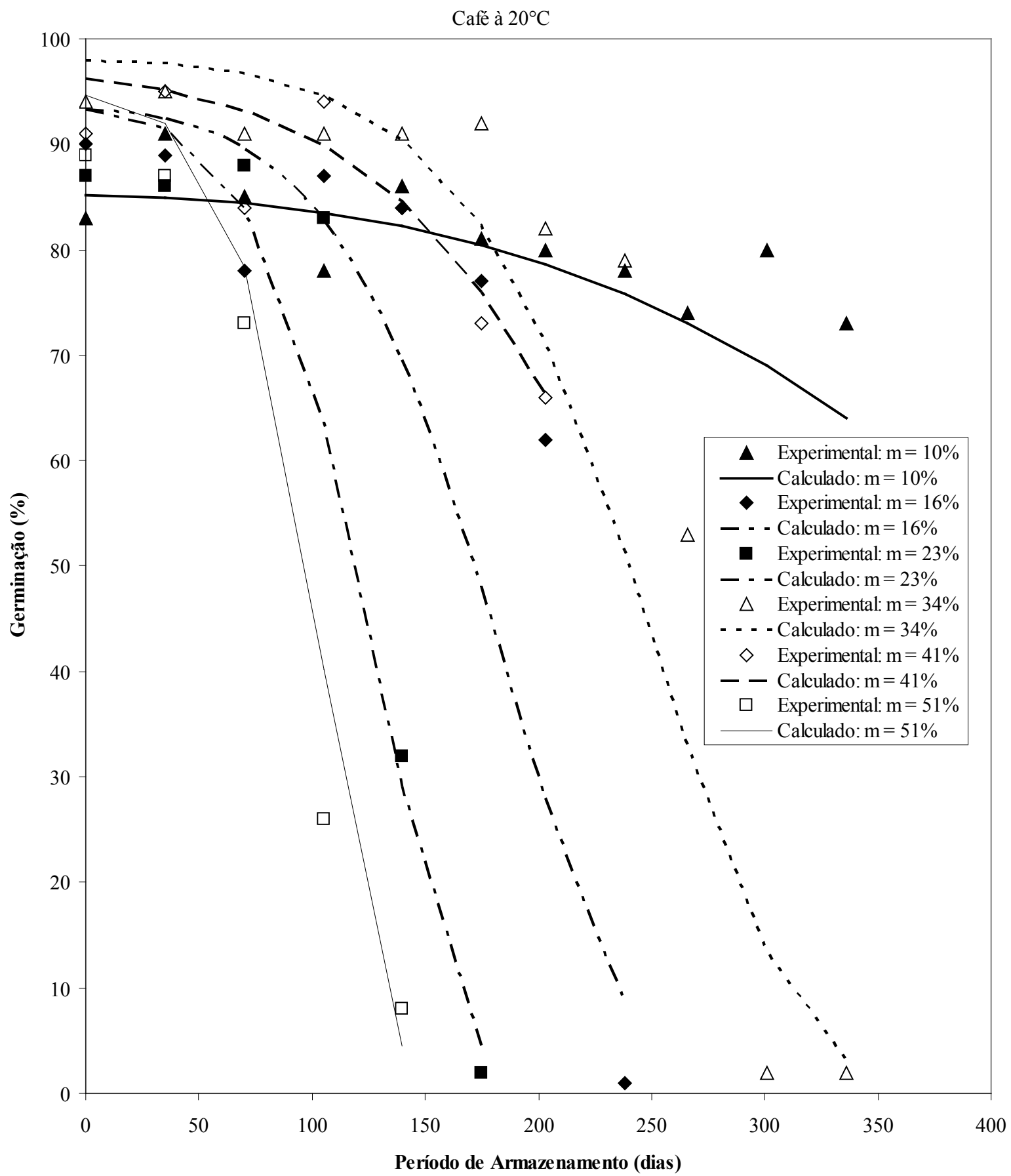

Figura 3. Valores experimentais e calculados (modelo proposto) da perda de germinação das sementes de café em função do período de armazenamento na temperatura de $20^{\circ} \mathrm{C}$ e teores de água de 10,16 , 23, 34, 41 e 51\% (dados experimentais de Gentil et al., 2001). 


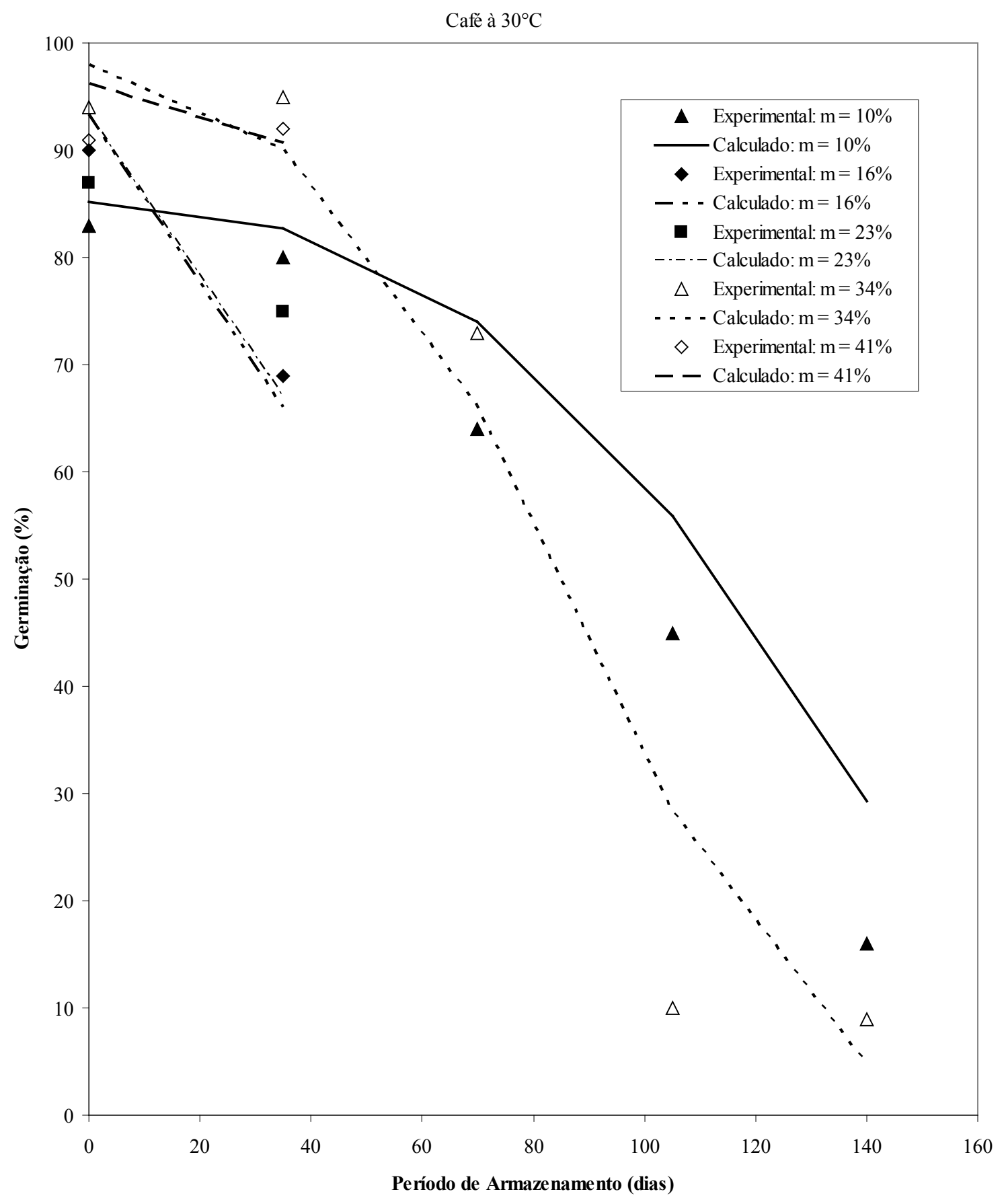

Figura 4. Valores experimentais e calculados (modelo proposto) da perda de germinação das sementes de café em função do período de armazenamento na temperatura de $30^{\circ} \mathrm{C}$ e teores de água de 10,16 , 23, 34 e 41\% (dados experimentais de Gentil et al., 2001). 


\section{CONCLUSÕES}

- O modelo quadrático apresentou ajuste adequado, com desvio padrão de $8,9 \%$ de germinação, para equacionar a perda de germinação de sementes de café armazenadas, enquanto o modelo tradicional apresentou um desvio padrão de $15,1 \%$ de germinação no equacionamento da perda de germinação de sementes de café armazenadas;

- A regressão não linear, comparada com a regressão linear múltipla, produziu menores desvios padrão (1,5 a 2,2 vezes) na determinação dos coeficientes da equação do desvio padrão da frequência de distribuição das sementes mortas durante o período de armazenamento $(\sigma)$;

- As sementes de café não apresentaram comportamento de ortodoxas, indicado pelas variações não lineares do logaritmo de $\sigma$ em função das variações do logaritmo do teor de água das sementes.

\section{REFERÊNCIAS BIBLIOGRÁFICAS}

BEWLEY, J.; BLACK, M. Seeds. Physiology of development and germination. $2^{\text {nd }}$ ed., Plenum Press, New York, 1994. 445p.

CARVALHO, N.M.; NAKAGAWA, J.; Sementes - ciência, tecnologia e produção. 4a $\mathrm{ed}$. Jaboticabal: FUNEP, 2000. 588p.

ELLIS, R.H.; ROBERTS, E. H. Improved equations for the prediction of seed longevity. Annals of Botany, Oxford, v.45, p.13-30, 1980.

ELLIS, R.H.; OSEI-BONSU, K.; ROBERTS, $\mathrm{H}$. The influence of genotype, temperature nd moisture on seed longevity in chickpea, cowpea and soybean. Annals of Botany, Oxford, v.50, p.69-82, 1982.

ELLIS, R.H.; HONG, T.D.; ROBERTS, E.H. An intermediate category of seed storage behaviour? I. Coffee. Journal of Experimental Botany, Oxford, v.41, n.230, p.1167- 1174, 1990.
FABRIZIUS, E.; TEKRONY, D.; EGLI, D. B.; RUCKER, M. Evaluation of a viability model for predicting soybean seed germination during warehouse storage. Crop Science, Madison, v.39, p.194-201, 1999.

FINNEY, D.J. Probit Analysis. $3^{\text {a }}$ ed., Cambridge University Press, London 1971.333p.

GENTIL, D.F.O.; SILVA, W.R.; MIRANDA, D.M. Grau de umidade e temperatura na conservação de sementes de café. Bragantia, Campinas, v.60, n.1, p.53-64, 2001.

GROOT, S.P.C.; SOEDA, Y.; STOOPEN, G.; KONINGS, M.C.J.M.; GEEST, A.H.M. van der. Gene expression during loss and regaining of stress tolerance at seed priming and drying. In: NICOLÁS, G.; BRADFORD, K.J.; CÔME, D.; PRITCHARD, H.D. (Ed.). The biology of seeds: recent research advances. Cambridge: $\mathrm{CAB}$ International, 2003, p.279-287.

HONG, T.D.; ELLIS, R.H. A protocol to determine seed storage behaviour.Rome: IPGRI, 1996. 62p. (IPGRI Technical Bulletin no. 1).

HONG, T.D., LININGTON, S., ELLIS, R.H. Seed Storage Behaviour: a Compendium. Handbooks for Genebanks: No. 4. International Plant Genetic Resources Institute, Rome, 1996. 120 p.

MEAD, A.; GRAY, D. Prediction of seed longevity: a modification of the shape of the Ellis and Roberts seed survival curves. Seed Science Research, Cambridge, v.9, p.63-73, 1999.

PIETA FILHO, C.; ELLIS, R.H. Estimating the value of the seed lot constant $(\mathrm{Ki})$ of the seed viability equation in barley and wheat. Seed Science and Technology, Zurich, v.20, p.93-99, 1992.

PRESS, W.H.; FLANNERY, B.P.; TEUKOLSKY, S.A.; VETTERLING, W.T. Numerical recipes. The art of scientific computing. Cambridge: Cambridge University Press, 1990. 874p. 
ROBERTS, E.H. Predicting the storage life of seeds. Seed Science and Technology, Zurich, v.1, p.499-514, 1973.

SAPRA, R.L.; NARAIM, P.; BHAT, S.R.; LAL, S.K.; JAIN, S.K. Prediction of seed longevity in the genebank: How reliable are the estimates? Current science, Bangalore, v.85, n.11, p.1612-1616, 2003.

SINÍCIO, R.; LOPES, J.F.; SILVA, D.J.H.; MATTEDI, A.P. Longevity equation for tomato seeds. Seed Science and Technology, Zurich, v.37, n.3, p.667-675, 2009.
SINÍCIO, R.; MAURI, A.L.; ARAUJO, E.F. Equacionamento da longevidade de sementes de tomate (Lycopersicum esculentum Mill). Revista Brasileira de Armazenamento, Viçosa, v.33, n.2, p.127-133, 2008.

USBERTI, R.; GOMES, R.B.R. Seed viability constants for groundnut. Annals of Botany, Oxford, v.82, p.691-694, 1998.

VERTUCCI, C.W.; ROOS, E.E. Theoretical bases of protocols for seed storage. Plant Physiology, Waterbury, v.94, p.1019-1023, 1990. 